\title{
A Facial Expression Recognition Method Based on Quantum Neural Networks
}

\author{
Peng Li Junhua Li \\ School of Communication and Control Engineering, Jiangnan University, Wuxi 214122, P. R. China
}

\begin{abstract}
The Facial Expression recognition is an important and complicated problem of pattern recognition field. In this paper, an approach to Facial Expression recognition based on multi-level transfer function Quantum Neural Networks (QNN) and multi-layer classifiers is presented. The QNN is trained and tested by the Japanese female facial expression (JAFFE) database. The experiment results indicate the method achieves excellent performance in terms of recognition rates and recognition reliability, and show the superiority and potential of QNN in solving pattern recognition problems.
\end{abstract}

Keywords: Quantum neural network, Multi-level transfer function, Multi-layer classifier, Facial expression recognition, Pattern recognition

\section{Introduction}

Facial expression recognition is significant foundation of human-computer interaction (HCI), involved with the fields of image processing, movement follow, patter recognition, physiology, psychology and so on. Facial expression recognition has attracted a significant interest in the patter recognition and artificial intelligence [1]. But, it is also a very defiant task because facial expression has relation to age, race, sex, illumination and so forth. And face is a gentle body, rather than just rigid body, and is hard to build up an ideal and precision facial expression model to describe.

In recent years, the artificial neural networks obtained to advance by leaps and bounds, putting forward many new methods. Facial expression recognition method based on neural networks is very valid because artificial neural networks has some attractive features such as parallel distributed processing, self-learning, fault tolerance and robustness and so forth. In various network models, the BP (Back propagation) network is the usage. But the BP network is the discretion boundary which adopts an upheaval to partition characteristic space, so BP network will can't the right estimate sample degree of membership in the cross of the characteristic space if The circumstance that the sample characteristic space's existence crosses [2]. Because the performance of facial expression includes many forms such as relaxative or excited, hairlike or intense, light-hearted or tensional and so on. This causes that the characteristic vectors of facial expression exist crossed and promiscuous. Therefore, the normal BP network recognition isn't ideal for facial expression recognition.

In this paper, an approach to Facial Expression recognition based on multi-level transfer function Quantum Neural Networks (QNN) and multi-layer classifiers is presented. The QNN is trained and tested by the CMU face expression image database. The experiment results indicate the method achieves excellent performance in terms of recognition rates and recognition reliability.

\section{The model of Quantum Neural Networks}

In 1997, N.B.Karayiannis and his colleagues introduced the model based on multi-level transfer function Quantum Neural Networks[3-5].Transfer function of neural cell in hidden layer adopts multilevel transfer function so as to network has the feature of fuzziness. It's proved that the model based on multi-level transfer function Quantum Neural Networks is useful in the indetermination and crossed pattern problem. The model based on multi-level transfer function Quantum Neural Networks has successfully applied to predict in the weather [6], voice recognition and disease diagnosis [4][7-8] and so forth.

Quantum neural network which is a multilayer feed forward neural network. It's effective to classify indeterminate data [4] [9-10]. The transfer function of quantum neural network express as linear superposition of multi-sigmoid function. It named multi-level transfer function [11]. In this way, a hidden layer neural cell can express more states than traditional sigmoid function which can express only two states. Each sigmoid function has different quantum interval. We can make different data map to different ladder by adjusting quantum interval for 
making classification have more freedom. The quantum interval of quantum neural network can obtain by training.

Quantum neural network can quantify indeterminate sampling data by learning arithmetic. The QNN will press certain comparison to assign it to all related categories if the characteristic vector of sample is the edge overlap which locates two types of modes. This make network have the feature of fuzziness and assign indeterminate data to all related categories. So the QNN decrease indeterminacy of pattern recognition and increase veracity of pattern recognition.

Quantum neural network classifier (Fig.1), where input vector is facial expression characteristic vector and output vector is expression classified vector.

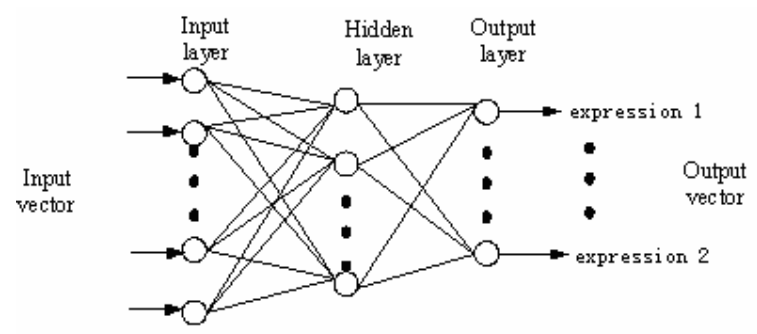

Fig. 1: The structure of Quantum Neural Network.

As to hidden cell's multi-layer transfer function of quantum neural network, the node of hidden layer output function is given as follows:

$$
b_{r}=\frac{1}{n s} \sum_{s=1}^{n s} f\left[\beta\left(W^{T} X-\theta_{s}\right)\right] \gamma=1,2, \cdots u
$$

where $f(x)=1 /(1+\exp (-x))$; $W$ is weight vector of network, $\boldsymbol{X}$ is input vector of network, $\beta$ is slope factor, $\mathbf{W}^{T} \mathbf{X}$ is input inspiriting of quantum cell , $\theta_{s}$ is quantum interval(s=1 $\left.\cdots n s\right)$.

The learning of quantum neural network includes two steps. The one is to adjust weight value for making input data map to different generic space. Another is to adjust quantum interval of hidden layer cell for expressing indeterminate data. We use BP arithmetic to adjust weight. Quantum interval is adjusted by corresponding arithmetic when we obtain weight. The thought of quantum interval adjusting is to make output change of hidden layer cell based on the same generic data minimize.

Suppose class $C_{m}$, the ith output of hidden layer cell is given as follows:

$$
\sigma_{i, m}^{2}=\sum_{x_{k}: x_{k} \in C_{m}}\left(\left\langle O_{i, m}\right\rangle-O_{i, k}\right)^{2}
$$

Where $O_{i, k}$ is the ith output of hidden layer cell when $x_{k}$ is input vector; $\left\langle O_{i, m}\right\rangle=\frac{1}{\left|C_{m}\right|} \sum_{x_{k}: x_{k} \in C_{m}} O_{i, k}$, $\left|C_{m}\right|$ is radix of $C_{m} \cdot \sigma_{i, m}^{2}$ is function of $\theta_{s}$ quantum interval. The derivative of $\theta_{s} \quad(\mathrm{~s}=1 \cdots \mathrm{ns})$ along equation (2) yields $\theta_{i, s}$ as follows:

$$
\begin{gathered}
\Delta \theta_{i, s}=\eta \frac{\beta}{n s} \sum_{m=1}^{n_{0}} \sum_{x_{k}: x_{k} \in C_{m}}\left(\left\langle O_{i, m}\right\rangle-O_{i, k}\right) *\left(\left\langle V_{i, m, s}\right\rangle-V_{i, k, s}\right) \\
\text { Where }\left\langle V_{i, m, s}\right\rangle=\frac{1}{\left|C_{m}\right|} \sum_{x_{k}: x_{k} \in C_{m}} V_{i, k, s} \\
V_{i, k, s}=O_{i, k, s} *\left(1-O_{i, k, s}\right)
\end{gathered}
$$

Where $O_{i, k, s}=\operatorname{sig}\left(\beta^{*}\left(w^{T} x_{k}-\theta_{s}\right)\right)$ is the sth output of the ith hidden layer cell when $x_{k}$ is input vector $(\mathrm{s}=1 \cdots \mathrm{ns})$. In equation (3), where $\eta$ is learning rate, $n_{0}$ is sum of output layer node namely, the sum of category; $n s$ is sum of quantum interval; $x_{k}: x_{k} \in C_{m}$ is all samples which belong to $C_{m}$.

\section{Facial expression recognition system based on quantum neural networks}

\subsection{Pretreatment}

In this paper, the QNN is trained and tested by the Japanese female facial expression database. This database currently contains 213 images of 7 facial expressions (6 basic facial expressions and 1 neutral) posed by 10 Japanese female models. Each image has been rated on 6 emotion adjectives by 60 Japanese subjects. A set of image in the facial expression database as follows:

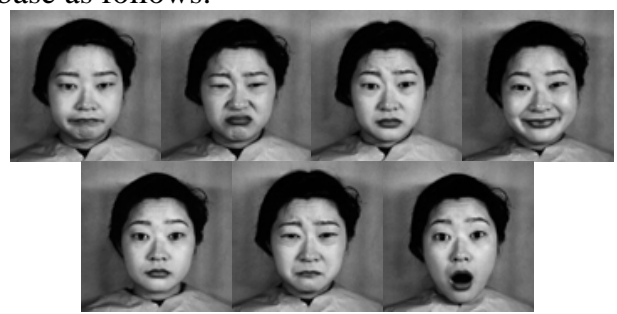


Fig. 2: The images of Japanese female facial expression (JAFFE) database.

We need pretreatment to eliminate noise such as figure, hair, illumination and so on before withdrawing characteristic vector. The pretreatment includes three steps as follows:

(1) Incising the original image to reserve the main characteristic district of face.

(2) The image after incising is processed by geometry convergence. The size is 64 by 64 pixels after processing the image.

(3) Illumination compensation.

Figure 3 (b), (c), (d) respectively is an original picture (a) after above 3 processing.

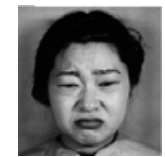

(a)

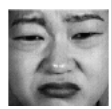

(b)

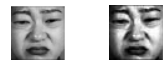

(c) (d)
Fig. 3: The pretreatment of facial expression image.

\subsection{The process of recognition system}

Recognition system work includes two parts of training process and recognition process. Training process is that QNN classifier learns by characteristic vector. We can obtain network weight, quantum interval when training attain target. And then quantum neural network use this network weight and quantum interval. We adopt batch conjugate grade method to update network weight. Recognition process is that QNN recognition system receives characteristic vector and export the result. The process of recognition is given in Figure 4.

The classifier receives characteristic vector and outputs the result, and then we choose dubious facial expression (R1, R2) by the result of the first classifier. These facial expressions (R1, R2) are inputted to the second classifier $\mathrm{v}$ (R1-R2).The facial expression is $\mathrm{R} 1$ if the output of second classifiers V (R1-R2) is all $\mathrm{R} 1$. The facial expression is $\mathrm{R} 2$ if the output of second classifiers V (R1-R2) is all R2. The result is rejected if the output isn't anterior circumstances. That will improve recognition rate.

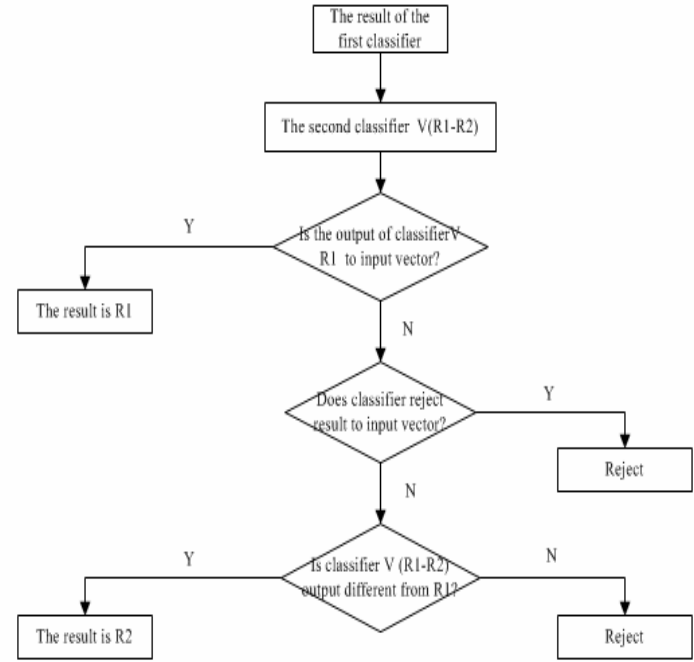

Fig. 4: The process of recognition system.

\section{Simulation and experimental result}

In this paper, the QNN is trained and tested by the Japanese female facial expression (JAFFE) database. The test database for the recognition consists of a total of 143 facial images selected from the JAFFE. Each person has 7 facial expression which is anger, disgust, fear, happiness, neutral, sadness and surprise. The rest is training samples. The first classifier will stop when square error is less than 0.002 ( $\mathrm{SSE} \leq 0.002$ ); The second classifier will stop when square error is less than 0.0005 (SSE $\leq$ 0.0005). The learning ratio of QNN weight and threshold both are 0.05, the learning ratio of quantum interval is 0.005 . We choose the slope factor of multi-level transfer function equal to the number of quantum interval, namely the first network slope factor is 6 , and the second network slope factor is 3. QNN classifier error is given in figure 5 and 6 .

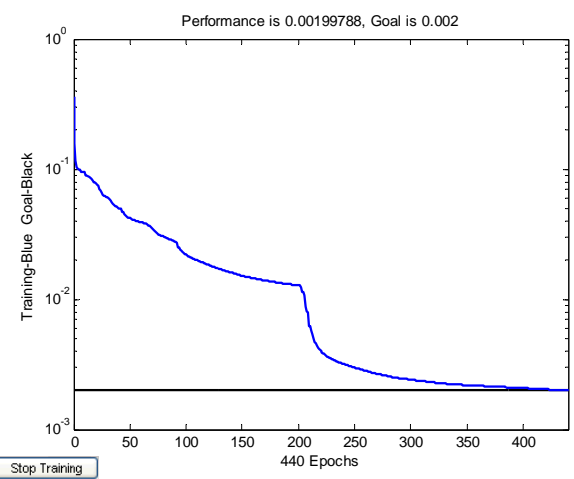

Fig. 5: The first QNNs classifier error. 


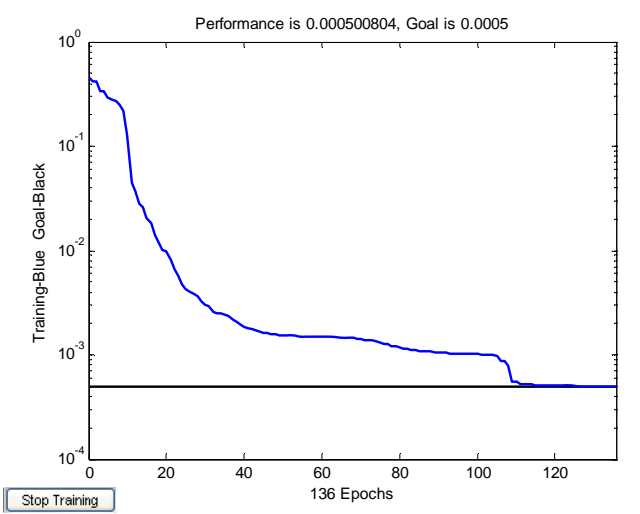

Fig. 6: The second QNNs classifier error.

We test the performance index of normal BP network, QNN classifier and QNN multi-layer classifiers recognition system. The result is given as follows:

\begin{tabular}{ccccc}
\hline Network & $\begin{array}{c}\text { Reject } \\
\text { rate }(\%)\end{array}$ & $\begin{array}{c}\text { Mistake } \\
\text { rate } \\
(\%)\end{array}$ & $\begin{array}{c}\text { rate } \\
(\%)\end{array}$ & $\begin{array}{c}\text { Reliability } \\
(\%)\end{array}$ \\
\hline $\begin{array}{c}\text { normal BP } \\
\text { network }\end{array}$ & 89.9 & 3 & 7.1 & 92.6 \\
$\begin{array}{c}\text { QNN } \\
\text { classifier }\end{array}$ & 91.7 & 4.9 & 3.4 & 96.4 \\
$\begin{array}{c}\text { QNN multi- } \\
\text { layer } \\
\text { classifiers }\end{array}$ & 96.5 & 2.3 & 1.2 & 98.8 \\
\hline
\end{tabular}

Table 1: The performance index of networks.

reliability $=$ Recognition rate / $($ Recognition rate + Mistake rate ) $\times 100 \%$

To demonstrate the effectiveness of QNN multilayer classifiers, experiments were performed on the JAFFE database. Its recognition rate and reliability both are obvious exaltation than other methods. Therefore, QNN multi-layer classifiers is good for facial expression recognition.

\section{Conclusion}

In this paper, an approach to Facial Expression recognition based on multi-level transfer function Quantum Neural Networks (QNN) and multi-layer classifiers is presented. The QNN is trained and tested by the Japanese female facial expression (JAFFE) database. Recognition rate reaches to $96.5 \%$. Reliability reaches to $98.8 \%$. The experiment results indicate the method achieves excellent performance. The future work is to find more discriminating features and more efficient method for facial expression recognition.

\section{References}

[1] M. Yeasin, B. Bullot and R. Sharma, Recognition of facial expressions and measurement of levels of interest from video. IEEE Transactions on Multimedia, pp.500-508, 2006.

[2] J. Zhou, Recognition and verification of unconstrained handwritten numerals. Canada: The Univ. of Concordia, 1999

[3] N.B. Karayiannis and Y.H. Xiong, Training reformulated radial basis function neural networks capable of identifying uncertainty in data classification. IEEE Transactions on Neural Networks, pp.1222-1234, 2006.

[4] J. Zhou, Q. Gan and A. Krzyzak, Recognition of handwritten numerals by quantum neural network with fuzzy features. International Journal on Document Analysis and Recognition, pp.30-36, 1999.

[5] E.C. Behman, V.G. Chandrashkar and C.K. Wang, A quantum neural network computes entanglement. Physical Review Letters, pp.152159, 2002.

[6] R. Kretzschmar, R. Bueler and N.B. Karayiannis, Quantum neural networks versus conventional feedforward neural networks: an experimental study. Proceedings of the 2000 IEEE Signal Processing Society Workshop, pp.328-337, 2000.

[7] N.B. Karayiannis, A. Mukherjee and J.R. Glover, An evaluation of quantum neural networks in the detection of epileptic seizures in the neonatal electroencephalogram. Soft Computing, pp.382396,2006.

[8] N.B. Karayiannis, A. Mukherjee and J.R. Glover, Detection of pseudosinusoidal epileptic segments in the neonatal EEG by cascading a rule -based algorithm with a neural network. IEEE Transaction on Biomedical Engineering, pp.633-641,2006.

[9] F. Li, S.G. Zhao and B.Y. Zheng, Quantum neural network in speech recognition. The 6th International Conference on Signal Processing, pp.1627-1270, 2002.

[10] E.C. Behrman, L.R. Nash and J.E. Steck, Simulations of quantum neural networks. Information Sciences, pp.257-269, 2000.

[11] S.D. Zhou, Y. Wang, Z.Q. Sun and F.C. Sun, China Intelligent Automatic Conference, pp.163168, 2003. 